\title{
Commutator Groups and Algebras*
}

\author{
L. Greenberg**
}

(March 12, 1969)

\begin{abstract}
Let $H$ and $K$ be connected, Lie subgroups of a Lie group $G$. The group $[H, K]$, generated by all commutators $h k h^{-1} k^{-1}(h \epsilon H, k \epsilon K)$ is arcwise connected. Therefore, by a theorem of Yamabe, $\lfloor H, K]$ is a Lie subgroup. If $\mathfrak{S}, \Re$ denote the Lie algebras of $H$ and $K$, respectively, then the Lie algebra of $[H, K]$ is the smallest algebra containing $[\mathfrak{S}, \Re]$, which is invariant under ad $\mathfrak{S}$ and $a d \Re$. An immediate consequence is that if $H$ and $K$ are complex Lie subgroups, then $[H, K]$ is also complex.
\end{abstract}

Key words: Adjoint representation; commutator; Lie algebra; Lie group.

Let $G$ be a real Lie group, and let $H$ and $K$ be connected, Lie subgroups, with Lie algebras $\mathfrak{h}$ and $\mathfrak{f}$. The group $[H, K]$ generated by commutators $\left\{h k h^{-1} k^{-1} \mid h \epsilon H, k \in K\right\}$ is arc-wise connected. This implies, by a theorem of Yamabe $[1],{ }^{1}$ that $[H, K]$ is a Lie subgroup. The question we raise in this note is: What is the Lie algebra of $[H, K]$ ? We shall prove that this is the smallest algebra which contains $[\mathfrak{h}, \mathfrak{f}]$ and is invariant under $a d \mathfrak{h}$ and $a d \mathfrak{f}$ (i.e., it is the smallest ideal in the algebra generated by $\mathfrak{h}$ and $\mathfrak{f}$ which contains $[\mathfrak{h}, \mathfrak{f}]$.) An immediate consequence is that if $H$ and $K$ are complex groups, then $[H, K]$ is also complex. Of course, one obtains as a special case the known fact that if $H$ and $K$ are normal, then $[\mathfrak{h}, \mathfrak{f}]$ is the Lie algebra of $[H, K]$. More generally, the Lie algebra of $[H, K]$ is the smallest algebra $m$ containing $[\mathfrak{h}, \mathfrak{f}]$, if and only if $m$ is invariant under $a d \mathfrak{h}$ and $a d \mathfrak{f}$.

We shall first give a list of notation and terminology. In the following, $G$ is a real Lie group, with Lie algebra g.

(1) If $g \epsilon G$ and $X$ is a tangent vector or vector field on $G$, then $g X$ and $X g$ denote the left and right translation of $X$. (i.e., if $l_{g}, r_{g}$ are the left and right translations of $G$, then $g X=d l_{g}(X)$ and $X g=d r_{g}(X)$.)

(2) We denote by $a d$ and $A d$ the adjoint representations of $g$ and $G$, respectively. Thus $a d(X) Y$ $=[X, Y]$ and $A d(g) Y=g Y g^{-1} . \theta(g)$ denotes the inner automorphism $x \rightarrow g x g^{-1}$. Note that

$$
A d(\exp X)=\exp (a d X) \text { and } \theta(g) \exp X=\exp [A d(g) X] .
$$

(3) If $X$ is a tangent vector at some point of $G, \hat{X}$ denotes the corresponding left-invariant vector field. Of course, $\mathfrak{g}$ is the algebra of left-invariant vector fields on $G$.

(4) If $X$ is a vector field, $g \in G$, then $X_{g}$ is the value of $X$ at $g$. If $\mathfrak{h} \subset g$ is a subalgebra, $\mathfrak{h}_{g}=\left\{X_{g} \mid X \epsilon \mathfrak{h}\right\}$.

(5) If $\mathfrak{h}$ and $\mathfrak{f}$ are subalgebras of $\mathfrak{g},[\mathfrak{h}, \mathfrak{f}]$ denotes the linear span of the commutators $\{[X, Y] \mid X \in \mathfrak{h}, Y \in \mathfrak{f}\}$.

(6) If $\mathfrak{m}$ is a subspace of $\mathfrak{g}, \mathscr{A} \mathfrak{m}$ denotes the subalgebra generated by $\mathrm{m}$.

(7) If $\mathfrak{m}$ is a subspace, $\mathfrak{h}$ a subalgebra, with $\mathfrak{m} \subset \mathfrak{h}$, then $\mathfrak{I}(\mathfrak{m}, \mathfrak{h})$ denotes the smallest ideal in $\mathfrak{h}$ which contains $\mathfrak{m}$. Thus $\mathfrak{I}(\mathfrak{m}, \mathfrak{h})$ is the linear span of $\mathfrak{m}$ and elements of the form $\left(a d X_{1}\right)\left(a d X_{2}\right) \ldots\left(a d X_{r}\right) Y$, where $X_{i} \in \mathfrak{h}, Y \in \mathfrak{m}$. We write $\mathfrak{I}[\mathfrak{h}, \mathfrak{f}]$ for $\mathfrak{I}([\mathfrak{h}, \mathfrak{f}], \mathscr{A}(\mathfrak{h}+\mathfrak{f}))$.

(8) If $g: I \rightarrow G$ is a differentiable curve (where $I$ is an interval), we write $\left.\frac{d g}{d t}\right|_{t_{0}}$ for the tangent vector $\left.d g\right|_{t_{0}}\left(\frac{d}{d t}\right)$. We shall write $0\left(\left(t-t_{0}\right)^{k}\right)$ for a function $X(t)$ (with values in $g$ ) if there exist numbers $M>0, \epsilon>0$ so that ||$X(t)||<M\left|t-t_{0}\right|^{k}$ when $\left|t-t_{0}\right|<\epsilon$. (Here ||$X||$ is some norm in g.)

\footnotetext{
*An invited paper. This work has been supported by National Science Foundation Grant NSF GP-5927.

**Present address: University of Maryland, Department of Mathematics, College Park, Md. 20740.

${ }^{1} \mathrm{H}$. Yamabe, On an arcwise connected subgroup of a Lie group, Osaka Math. J. 2, 13-14 (1950).
} 
For example, if $g(t)$ is a differentiable curve in $G$, with $g\left(t_{0}\right)=1$ and $\frac{d g}{d t} \mid t_{0}=X$, then (for $t$ near $\left.t_{0}\right) \log [g(t)]=\left(t-t_{0}\right) X+0\left(\left(t-t_{0}\right)^{2}\right)$, or equivalently, $g(t)=\exp \left[\left(t-t_{0}\right) X+0\left(\left(t-t_{0}\right)^{2}\right)\right]$. The Campbell-Baker-Hausdorff formula implies.

$$
\exp (t X) \exp (t Y)=\exp \left(t(X+Y)+\frac{t^{2}}{2}[X, Y]+0\left(t^{3}\right)\right) .
$$

LEMMA 1: Let $\mathrm{H}$ and $\mathrm{K}$ be connected Lie subgroups of $\mathrm{G}$, with Lie algebras $\mathfrak{h}$ and $\mathfrak{f}$.

(a) If $\mathrm{h} \epsilon \mathrm{H}$ and $\mathrm{Y} \in \mathfrak{f}$, then $(\operatorname{Ad}(\mathrm{h})-1) \mathrm{Y} \in \mathfrak{J}[\mathfrak{h}, \mathfrak{f}]$.

(b) If $\mathrm{k} \in \mathrm{K}$ and $\mathrm{X} \in \mathfrak{h}$, then $(\operatorname{Ad}(\mathrm{k})-1) \mathrm{X} \epsilon \mathfrak{I}[\mathfrak{h}, \mathfrak{f}]$.

Proof: We shall prove (a). Note that $\left(A d\left(h_{1} h_{2}\right)-1\right) Y=A d\left(h_{1}\right)\left(A d\left(h_{2}\right)-1\right) Y+\left(A d\left(h_{1}\right)-1\right) Y$.

Thus, if the statement is true for $h_{1}$ and $h_{2}$, it is true for $h_{1} h_{2}$. Therefore it suffices to prove it for a set of generators. We shall show that it is true for $h=\exp (X), X \in \mathfrak{h}$.

$$
A d(\exp X)-1=\exp (a d X)-1=\sum_{n=1}^{\infty} \frac{(a d X)^{n}}{n !}
$$

while $(a d X)^{n} Y \epsilon \mathfrak{I}[\mathfrak{h}, \mathfrak{f}]$.

Q.E.D.

LEMmA 2: Let $\mathrm{H}$ and $\mathrm{K}$ be connected, Lie subgroups of $\mathrm{G}$, with Lie algebras $\mathfrak{h}$ and $\mathfrak{f}$. Let $\mathrm{h}(\mathrm{t})$ (respectively $\mathrm{k}(\mathrm{t}))$ be a differentiable curve in $\mathrm{H}$ (respectively $\mathrm{K})$ with $\mathrm{h}(0)=1$ (respectively $\mathrm{k}(0)=1)$. Let $\mathrm{g}(\mathrm{t})=\mathrm{h}(\sqrt{\mathrm{t}}) \mathrm{k}(\sqrt{\mathrm{t}}) \mathrm{h}(\sqrt{\mathrm{t}})^{-1} \mathrm{k}(\sqrt{\mathrm{t}})^{-1}$. Then for each $\mathrm{t}_{0} \geqslant 0$ at which $\mathrm{g}(\mathrm{t})$ is defined,

$$
\left.\frac{\mathrm{dg}}{\mathrm{dt}}\right|_{\mathrm{t}_{0}} \in \mathfrak{\Im}[\mathfrak{h}, \mathfrak{f}]_{\mathrm{g}\left(\mathrm{t}_{0}\right)} .
$$

Proof: For $t_{0}=0,\left.\frac{d g}{d t}\right|_{0}=[\hat{X}, \hat{Y}]_{1}$, where $X=\left.\frac{d h}{d t}\right|_{0}$ and $Y=\left.\frac{d k}{d t}\right|_{0}$. We must find out what happens when $t_{0}>0$.

Let $u=\sqrt{t}, u_{0}=\sqrt{t_{0}}, h_{0}=h\left(u_{0}\right), k_{0}=k\left(u_{0}\right)$,

$$
\begin{aligned}
& g_{0}=g\left(t_{0}\right)=h_{0} k_{0} h_{0}^{-1} k_{0}^{-1}, \\
& X=\left.\frac{d h(u)}{d u}\right|_{u_{0}}, Y=\left.\frac{d k(u)}{d u}\right|_{u_{0}} \text { and } Z=\left.\frac{d g(t)}{d t}\right|_{t_{0}}
\end{aligned}
$$

The translated curve $g_{0}^{-1} g(t)$ has tangent vector $g_{0}^{-1} Z$ at 1 . We shall use properties of exp to compute this tangent vector.

Noting that

$$
\left.\frac{d}{d u}\left(h_{0}^{-1} h(u)\right)\right|_{u_{0}}=h_{0}^{-1} X
$$

and

$$
\left.\frac{d}{d u}\left(h_{0} h(u)^{-1}\right)\right|_{u_{0}}=-\left.\frac{d}{d u}\left(h(u) h o^{-1}\right)\right|_{u_{0}}=-X h_{0}^{-1}
$$

We obtain

(1) $h_{0}^{-1} h(u)=\exp \left\{\left(u-u_{0}\right) \hat{X}+0\left(\left(u-u_{0}\right)^{2}\right)\right\}$,

Similarly,

(2) $h_{0} h(u)^{-1}=\exp \left\{-\left(u-u_{0}\right) A d\left(h_{0}\right) \hat{X}+0\left(\left(u-u_{0}\right)^{2}\right)\right\}$.

(3) $k(u) k_{0}^{-1}=\exp \left\{\left(u-u_{0}\right) A d\left(k_{0}\right) \hat{Y}+0\left(\left(u-u_{0}\right)^{2}\right)\right\}$,

(4) $k(u)^{-1} k_{0}=\exp \left\{-\left(u-u_{0}\right) \hat{Y}+0\left(\left(u-u_{0}\right)^{2}\right)\right\}$.

Next, we see that

$$
\begin{aligned}
g_{0}^{-1} g(t) & =k_{0} h_{0} k_{0}^{-1} h_{0}^{-1} h(u) k(u) h(u)^{-1} k(u)^{-1} \\
& =\theta\left(k_{0}\right)\left\{\left[\theta\left(h_{0} k_{0}^{-1}\right)\left(h_{0}^{-1} h(u) k(u) k_{0}^{-1}\right)\right]\left[h_{0} h(u)^{-1} k(u)^{-1} k_{0}\right]\right\} .
\end{aligned}
$$


Now by using equations (1)-(4),

(5) $\exp \left(s U+0\left(s^{2}\right)\right) \exp \left(s V+0\left(s^{2}\right)\right)=\exp \left\{s(U+V)+0\left(s^{2}\right)\right\}$

and

(6) $\theta(g) \exp (U)=\exp [A d(g) U]$,

a straightforward computation shows that $g_{0}^{-1} g(t)=\exp [W(t)]$, where

$$
W(t)=\left(u-u_{0}\right)\left\{A d\left(k_{0} h_{0}\right)\left(A d\left(k_{0}^{-1}\right)-1\right) \hat{X}+A d\left(k_{0}\right)\left(A d\left(h_{0}\right)-1\right) \hat{Y}\right\}+0\left(\left(u-u_{0}\right)^{2}\right) .
$$

It follows that the tangent vector $g_{0}^{-1} Z$ is the value of the vector field

$$
\left.\frac{d W}{d t}\right|_{t_{0}}=\frac{1}{\sqrt{t_{0}}}\left\{A d\left(k_{0} h_{0}\right)\left(A d\left(k_{0}^{-1}\right)-1\right) \hat{X}+A d\left(k_{0}\right)\left(A d\left(h_{0}\right)-1\right) \hat{Y}\right\}
$$

at the unit element. Thus, by Lemma $1, g_{0}^{-1} Z \epsilon \mathfrak{I}[\mathfrak{h}, \mathfrak{f}]_{1}$ and $Z \epsilon \mathfrak{I}[\mathfrak{h}, \mathfrak{f}]_{g_{0}}$.

Lemma 3: $[\mathrm{H}, \mathrm{K}]$ is normalized by $\mathrm{H}$ and $\mathrm{K}$.

Q.E.D.

Proof: Let $h$ and $h_{1} \in H, k \in K$. Then

$$
h_{1}\left(h k h^{-1} k^{-1}\right) h_{1}^{-1}=\left(h_{1} h k h^{-1} h_{1}^{-1} k^{-1}\right)\left(k h_{1} k^{-1} h_{1}^{-1}\right) .
$$

This shows that $[H, K]$ is normalized by $H$. Since $[H, K]=[K, H]$, the same is true for $K$.

Q.E.D.

Theorem: Let $\mathrm{G}$ be a real Lie group, and let $\mathrm{H}$ and $\mathrm{K}$ be connected, Lie subgroups with Lie algebras $\mathfrak{h}$ and $\mathfrak{f}$. Then $[\mathrm{H}, \mathbf{K}]$ is a Lie subgroup with Lie algebra $\Im[\mathfrak{h}, \mathfrak{f}]$.

Proof. We have already remarked that Yamabe's theorem implies that $[H, K]$ is a Lie subgroup. Let $m$ denote its Lie algebra. We first show that $\mathfrak{I}[\mathfrak{h}, \mathfrak{f}] \subset \mathrm{m}$. If $X \in \mathfrak{h}, Y \in \mathfrak{f}$, then the curve

$$
g(t)=\exp (\sqrt{t X}) \exp (\sqrt{t Y}) \exp (-\sqrt{t X}) \exp (-\sqrt{t Y})
$$

lies in $[H, K]$. Its tangent vector at 1 is $[X, Y]_{1}$. This shows that $[\mathfrak{h}, \mathfrak{f}] \subset \mathrm{m}$. Lemma 3 implies that $m$ is invariant under $\operatorname{ad}(\mathfrak{h})$ and $\operatorname{ad}(\mathfrak{f})$. Therefore $\mathfrak{J}[\mathfrak{h}, \mathfrak{f}] \subset \mathrm{m}$.

Now we show that $\mathfrak{m} \subset \Im[\mathfrak{h}, \mathfrak{f}]$. Equivalently, we show that $[H, K] \subset L$, where $L$ is the connected Lie subgroup whose Lie algebra is $\mathfrak{I}[\mathfrak{h}, \mathfrak{f}]$. Let $h \epsilon H, k \epsilon K$, and let $h(t)$ and $k(t)$ be differentiable curves in $H$ and $K$, respectively, so that $h(0)=1=k(0), h(1)=h$ and $k(1)=k$. Let

$$
g(t)=h(\sqrt{t}) k(\sqrt{t}) h(\sqrt{t})^{-1} k(\sqrt{t})^{-1} .
$$

Lemma 2 says that

$$
\left.\frac{d g}{d t}\right|_{t_{0}} \epsilon \mathfrak{I}[\mathfrak{h}, \mathfrak{f}]_{g\left(t_{0}\right)} \quad\left(0 \leqslant t_{0} \leqslant 1\right) .
$$

Therefore, the curve $g(t)$ lies in the maximal connected integral manifold (through 1 ) of the differential system $\mathfrak{\Im}[\mathfrak{h}, \mathfrak{f}]$. In other words, the curve $g(t)$ lies in $L$. Thus, the commutators $h k h^{-1} k^{-1} \epsilon L$, and consequently $[H, K] \subset L$.

Q.E.D.

Corollary 1: Let $\mathrm{G}$ be a complex Lie group, and let $\mathrm{H}$ and $\mathrm{K}$ be connected (complex) Lie subgroups. Then $[\mathrm{H}, \mathrm{K}]$ is a (complex) Lie subgroup.

Proof: Considering $G$ with its real structure, we see that $[H, K]$ is a real Lie subgroup whose Lie algebra is $\mathfrak{I}[\mathfrak{h}, \mathfrak{f}]$ (where $\mathfrak{h}$ and $\mathfrak{f}$ are the Lie algebras of $H$ and $K$ ). Since $\mathfrak{h}$ and $\mathfrak{f}$ are complex, so is $\mathfrak{I}[\mathfrak{h}, \mathfrak{f}]$ and $[H, K]$.

Q.E.D.

Corollary 2: The Lie algebra of $[\mathrm{H}, \mathrm{K}]$ is $\mathscr{A}[\mathfrak{h}, \mathfrak{f}]$, if and only if $\mathscr{A}[\mathfrak{h}, \mathfrak{f}]$ is invariant under $\operatorname{ad}(\mathfrak{h})$ and $\operatorname{ad}(\mathfrak{f})$.

(Paper 73B3-305) 